\title{
Forecasting the Spanish economy with an augmented VAR-DSGE model
}

\author{
Gonzalo Fernández-de-Córdoba · José L. Torres
}

Received: 14 May 2009 / Accepted: 24 March 2010 / Published online: 1 March 2011

(C) The Author(s) 2011. This article is published with open access at SpringerLink.com

\begin{abstract}
Over the past 10 years dynamic stochastic general equilibrium (DSGE) models have become an important tool in quantitative macroeconomics. However, DSGE models were not considered as a forecasting tool until very recently. The objective of this paper is twofold. First, we compare the forecasting ability of a canonical DSGE model for the Spanish economy with other standard econometric techniques. More precisely, we compare out-of-sample forecasts obtained from different estimation methods of the DSGE model with the forecasts produced by a VAR and a Bayesian VAR. Second, we propose a new method for combining DSGE and VAR models (in what we have called Augmented VAR-DSGE) through the expansion of the variable space where the VAR operates with artificial series obtained from a DSGE model. The results indicate that the out-of-sample forecasting performance of the proposed method is capable of competing with all the considered alternatives, and thus even a simple canonical RBC model contains useful information that can be used for forecasting purposes.
\end{abstract}

Keywords DSGE models $\cdot$ Forecasting $\cdot$ VAR $\cdot$ BVAR

JEL Classification $\quad$ C53 $\cdot$ E32 $\cdot$ E37

We would like to thank J. Pérez and two anonymous referees for very useful comments and suggestions. The authors acknowledge financial support from SEJ-122 and Junta de Andalucía-Proyecto de Excelencia P07-SEJ-02479.

G. Fernández-de-Córdoba · J. L. Torres $(\varangle)$

Departamento de Teoría e Historia Económica, Universidad de Málaga,

Campus El Ejido s/n, 29013 Málaga, Spain

e-mail: jtorres@uma.es 


\section{Introduction}

Forecasting macroeconomic variables is a crucial issue for both practitioners and policymakers, since the decisions of the former are based on the forecasts of key macroeconomic time series made by the latter. As pointed out by Litterman (1986a,b) economic forecasting is a very difficult task for several reasons: there is only a limited amount of data, available data often contain major measurement errors and relationships between economic variables are complex. However, for both central banks and governments, forecasting is a key element in the design and implementation of economic policies. Therefore, theoretical models must be designed in order to be a useful tool not only for policy analysis but also for forecasting.

Following Diebold (1998) macroeconomics forecasting follows two distinct approaches: structural and non-structural forecasting methods. Non-structural macroeconomic forecasting methods attempt to exploit the reduced-form correlations between macroeconomic variables, while structural macroeconomic forecasting is grounded on economic theory. According to Diebold (1998), the failure of large-scale macroeconomic forecasting models and Lucas (1976) critique led to the abandon of the structural macroeconometrics approach and to the dramatic growth of non-structural econometric forecasting methods in the 1970s. However, despite its importance, the performance of macroeconomic forecasting has shown important failures in both, the structural and non-structural approaches.

After the empire of the Box-Jenkins methodology, and following Sims (1980) and Litterman (1986a,b) the use of (Bayesian) vector autoregressive (VAR and BVAR) time series to forecast key macroeconomic variables became standard. The advantages of VARs and BVARs are multiple: they are easy to estimate, generate out-of-sample forecasts, and are very flexible. VAR models thus became very popular in the macroeconomists' toolbox. However, they present no (unrestricted VARs) or little (Structural VARs) economic theory.

The alternative approach to purely statistical methods is structural forecasting, using a theory-based approach. However, the traditional Keynesian structural forecasting approach went into declined in the 1970s due to the effects of the Lucas critique. The resurgence of structural macroeconomic forecasting was based on dynamic stochastic general equilibrium (DSGE) modelling developments. Today DSGE models have become the most popular tool in quantitative macroeconomics. In the 1990's and especially in the last 10 years, we have witnessed to an impressive developments in the specification and empirical application of DSGE models, which have become a laboratory for macroeconomists and the standard tool for policy analysis. ${ }^{1}$ DSGE models have a strong theoretical background as they are firmly grounded on modern micro-foundations. In the last few years, DSGE models have increased considerably

\footnotetext{
1 Most central banks and other public institutions have recently developed DSGE models. Representative examples are the Sveriges Riksbank (RAMSES) model, developed by Adolfson et al. (2007), the New Area-Wide model (NAWM) developed at the European Central Bank by Christoffel et al. (2008), the model developed at the Federal Reserve Board by Edge et al. (2008) and, the SIGMA model by Erceg et al. (2006), among others.
} 
in complexity, and size, incorporating several types of rigidity emphasized by the New Keynesian literature. ${ }^{2}$

The increasing number and size of the models recently developed account for the success of this methodology. The REMS model by Boscá et al. (2009); the MEDEA model by Burriel et al. (2009); the BEMOD model by Andrés et al. (2006) are just some of the DSGE developed recently for the Spanish economy.

However, despite their success DSGE models were not considered as a forecasting tool until very recently. As pointed out by Smets and Wouters (2003, 2005), DSGE models were only rarely applied to forecasting. The seminal works of Smets and Wouters $(2003,2005)$ lead to an emergent literature studying the forecasting performance of DSGE models compared to alternative non-structural models.

In general, DSGE forecasting entails the estimation of a hybrid model that combines theoretical DSGE models with the flexibility of atheoretical VAR models. ${ }^{3}$ Different methods for solving, estimating and forecasting with DSGE models have been proposed in the literature: Sargent (1989) and Altug (1989) proposed augmenting a DSGE model with measurement error terms following a first order autoregressive process, known as the DSGE-AR approach. Ireland (2004) proposed a method that is similar to that of Sargent (1989) and Altug (1989) but imposing no restriction on the measurement errors, assuming that residuals follow a first-order vector autoregression. We refer to this method as the DSGE-VAR approach.

An alternative, and somewhat different, approach is the one proposed by DeJong et al. (1993) and Ingram and Whiteman (1994) and further developed by Del Negro and Schorfheide (2003, 2004). They proposed the use of general equilibrium models as priors for Bayesian VARs. We refer to this method as the VAR-DSGE approach. ${ }^{4}$ DeJong, Ingram and Whiteman (1993) and Ingram and Whiteman (1994) developed a strategy for improving time series forecasts by shrinking vector autoregression coefficient estimates given a prior derived from a DSGE model. They showed that a simple DSGE model can improve the forecasting performance of an unrestricted VAR. However, they also reported that the forecasting performance of the VAR-DSGE was similar to that of a Bayesian VAR with the Minnesota prior.

The objective of this paper is twofold. First, this paper places in a forecasting competition the structural approaches we have already referred to, through a small scale canonical RBC model (using DSGE-AR, DSGE-VAR and VAR-DSGE methods) with the standard non-structural VAR and BVAR methods. The canonical RBC model was chosen for several reasons, following the lines of Diebold (1998): The scale of DSGE models has consistently grown over time and New Keynesian features have been incorporated that have improved our understanding of key macroeconomics variables underlying dynamics. However, for forecasting purposes the simplicity

\footnotetext{
2 Examples of these New Keynesian DSGE models are Smets and Wouters (2003) and Christiano et al. (2005).

3 See Canova (2002) for a comparison of the quantitative implications of DSGE models with those of unconstrained VAR models.

4 Some authors, as Del Negro and Schorfheide (2003), call this method the DSGE-VAR procedure. However, in order to avoid confusion with the method proposed by Ireland (2004), also called DSGE-VAR method in the literature, we will refer to it as the VAR-DSGE procedure.
} 
of the model could be an advantage. Therefore, before incorporating New Keynesian features we would like to make a forecasting assessment within a simple DSGE model using alternative techniques.

In addition, we propose a new approach consisting of the expansion of the variables space where the VAR operates with the addition of artificial series obtained from a carefully calibrated dynamic general equilibrium model, as an alternative strategy of combining DSGE and VAR models. This new approach is simple, powerful and easy to implement empirically. We show an example of how this approach can be implemented taking the canonical RBC model as a reference. When solving a DSGE model, we obtain time series for unobservable variables with a clear theoretical interpretation that are relevant in explaining the dynamics of the economy. If the specification of the model is a good approximation of the underlying relations between the macroeconomic variables that we are interested in, those unobserved variables contain information about the observable macroeconomic variables. Moreover, the VAR can be augmented not only with unobserved variables but also with observed ones, such as the stock of capital. In general, this approach can be interpreted as a new technique for mixing structural forecasting methods through DSGE models with standard nonstructural forecasting methods such as VAR and BVAR models. We refer to this new procedure as the Augmented (B)VAR-DSGE approach.

The exercise is conducted for the Spanish economy, focusing on forecasting four key macroeconomic variables: output, consumption, investment and labor, for the period 1980:1-2007:4. The ex post forecast errors are evaluated on the basis of the data from the period 1995:1-2007:4. The results indicate that the out-of-sample forecasting performance of the proposed Augmented (B)VAR model competes well with the alternatives considered. The AVAR model outperforms an unrestricted VAR model and the ABVAR model also outperforms the BVAR model. Thus we find that even a simple canonical RBC model contains useful information that can be used for forecasting purposes. We also find that the proposed method outperforms the other structural approaches. The only alternative with a similar forecasting performance is the DSGE-AR model.

The structure of the rest of the paper is as follows. In Sect. 2 we describe the theoretical DSGE model. The solution and estimation of the model are laid out in Sect. 3. Section 4 describes the alternative method proposed here to combine DSGE and VAR models. Section 5 describes the data, calibration and estimation of the different models. Section 6 presents some statistics to determine the relative performances of the different models. Section 7 concludes with final comments.

\section{The model}

In this section we describe the prototype DSGE model used in the rest of the paper. The scale of DSGE models has grown over time, specially in the last 10 years, with the incorporation of a large number of New Keynesian features. However, as pointed out by Diebold (1998) the scale of DSGE models must be as small as possible for two reasons. First, the demise of large-scale macroeconomics models has shown that bigger models are not necessarily better. Second, DSGE models requires their parameters 
to be jointly estimated, which implies a limitation on the complexity of these models. On the other hand, the final objective of the paper is to compare the forecasting performance of DSGE based alternative approaches with non-structural models. Therefore, for this comparative exercise we consider the use of a canonical RBC model to be more suitable.

\subsection{Households}

Consider a representative consumer whose preferences are represented by the following instantaneous utility function:

$$
U\left(C_{t}, N_{t} \bar{H}-L_{t}\right)=\gamma \log C_{t}+(1-\gamma) \log \left(N_{t} \bar{H}-L_{t}\right)
$$

Private consumption is denoted by $C_{t}$. Leisure, $N_{t} \bar{H}-L_{t}$, is calculated as the number of effective hours in the week times the number of weeks in a year $\bar{H}$, times the population at the age of taking labor-leisure decisions, $N_{t}$, minus the aggregated number of hours worked in a year $L_{t}$. The parameter $\gamma(0<\gamma<1)$ is the proportion of private consumption to total private income. The budget constraint faced by the representative consumer is:

$$
\left(1+\tau_{t}^{c}\right) C_{t}+K_{t}-K_{t-1}=\left(1-\tau_{t}^{l}\right) W_{t} L_{t}+\left(1-\tau_{t}^{k}\right)\left(R_{t}-\delta\right) K_{t-1}+T_{t},
$$

where $T_{t}$ is the transfer received by consumers from the government, $K_{t}$ is private capital stock, $W_{t}$ is the compensation to employees, $R_{t}$ is the rental rate, $\delta$ is the capital depreciation rate which is modelled as tax deductible, and $\tau_{t}^{c}, \tau_{t}^{l}, \tau_{t}^{k}$, are the private consumption tax, the labor income tax, and the capital income tax, respectively. The budget constraint indicates that consumption and investment cannot exceed income (net of taxes) and lump sum transfers.

The representative consumer maximizes the value of his lifetime utility given by:

$$
\underset{\left\{C_{t}, L_{t}\right\}_{t=0}^{\infty}}{\operatorname{Max}} E\left[\sum_{t=0}^{\infty} \beta^{t}\left[\gamma \log C_{t}+(1-\gamma) \log \left(N_{t} \bar{H}-L_{t}\right)\right]\right]
$$

subject to the budget constraint given $\tau_{t}^{c}, \tau_{t}^{l}, \tau_{t}^{k}$ and $K_{0}$, where $\beta \in(0,1)$, is the consumer's discount factor.

\subsection{Firms}

The problem of the firm is to find optimal values for the utilization of labor and capital. The production of final output, $Y_{t}$, requires labor services, $L_{t}$, and capital, $K_{t}$. Goods and factors markets are assumed to be perfectly competitive. The firm rents capital and hires labor to maximize period profits, taking factor prices as given. The technology exhibits a constant return to private factors and thus the profits are zero in equilibrium. 
The technology used by the firm is given by:

$$
Y_{t}=A_{t} K_{t-1}^{\alpha} L_{t}^{1-\alpha}
$$

where $A_{t}$ is a measure of total factor productivity and $\alpha$ is the capital share of output. The technology shock $A_{t}$ is assumed to follow a first-order autoregressive process:

$$
\ln \left(A_{t}\right)=\left(1-\rho_{A}\right) \ln (\bar{A})+\rho \ln \left(A_{t-1}\right)+\varepsilon_{t}
$$

where $\bar{A}>0, \rho_{A}<1$, and $\varepsilon_{t} \sim N\left(0, \sigma^{2}\right)$.

\subsection{Government}

The government uses tax revenues to finance spending trough lump-sum transfers paid out to consumers. We assume that the government balances its budget period-byperiod by returning revenues from distortionary taxation to the agents via lump-sum transfers, $T_{t}$. The government budget in each period is given by,

$$
\tau_{t}^{c} C_{t}+\tau_{t}^{l} W_{t} L_{t}+\tau_{t}^{k}\left(R_{t}-\delta\right) K_{t-1}=T_{t}
$$

\subsection{Equilibrium}

The model has implications for six variables: $Y_{t}, C_{t}, I_{t}, K_{t}, L_{t}$, and $A_{t}$. The parameters of the model are: $\alpha, \beta, \gamma, \delta, \bar{A}, \rho, \sigma$, the three tax rates, $\tau_{t}^{c}, \tau_{t}^{l}, \tau_{t}^{k}$, and the Lagrange multiplier $\lambda_{t}$. The first-order conditions for the consumer are:

$$
\begin{array}{r}
\gamma \frac{1}{C_{t}}-\lambda_{t}\left(1+\tau_{t}^{c}\right)=0, \\
-(1-\gamma) \frac{1}{N_{t} \bar{H}-L_{t}}+\lambda_{t}\left(1-\tau_{t}^{l}\right) W_{t}=0, \\
\beta\left[\lambda_{t}\left(1+\left(1-\tau_{t}^{k}\right)\left(R_{t}-\delta\right)\right)\right]-\lambda_{t-1}=0 .
\end{array}
$$

Combining (5) and (6) we obtain the condition that equates the marginal rate of substitution between consumption and leisure, i.e., the opportunity cost in terms of consumption (the numeraire) of an additional hour of leisure:

$$
\frac{1-\gamma}{\gamma} \frac{\left(1+\tau_{t}^{c}\right) C_{t}}{N_{t} \bar{H}-L_{t}}=\left(1-\tau_{t}^{l}\right) W_{p, t}
$$

Combining (5) and (7) gives

$$
\frac{1}{\beta} \frac{C_{t+1}}{C_{t}}=\left(1-\tau_{t+1}^{k}\right) R_{t+1}+(1-\delta),
$$


The first-order conditions from the firm's maximization problem are:

$$
\begin{aligned}
R_{t} & =\alpha A_{t} K_{t-1}^{\alpha-1} L_{t}^{1-\alpha}, \\
W_{t} & =(1-\alpha) A_{t} K_{t-1}^{\alpha} L_{t}^{-\alpha},
\end{aligned}
$$

Thus, the economy satisfies the following feasibility constraint:

$$
C_{t}+I_{t}=R_{t} K_{t-1}+W_{t} L_{t}=Y_{t}
$$

where investment enters in the permanent inventory equation of capital accumulation as,

$$
I_{t}=K_{t}-(1-\delta) K_{t-1}
$$

Together with the first-order conditions of the firm, the budget constraint of the government (4), and the feasibility constraint of the economy, (12), characterize a competitive equilibrium for the economy.

\section{Solving the DSGE model}

Our model has six variables $\left(Y_{t}, C_{t}, I_{t}, L_{t}, K_{t}\right.$, and $\left.A_{t}\right)$ and the equilibrium behaviour of the economy is determined by the following six equations:

$$
\begin{aligned}
Y_{t} & =A_{t} K_{t-1}^{\alpha} L_{t}^{1-\alpha} \\
Y_{t} & =C_{t}+I_{t} \\
K_{t} & =(1-\delta) K_{t-1}+I_{t} \\
\frac{1}{C_{t}} & =\beta E_{t}\left[\frac{1}{C_{t+1}}\left(1+\left(1-\tau^{k}\right)\left(\alpha \frac{Y_{t+1}}{K_{t+1}}-\delta\right)\right)\right] \\
\frac{1-\gamma}{\gamma} \frac{\left(1+\tau^{c}\right) C_{t}}{N_{t} \bar{H}-L_{t}} & =\left(1-\tau^{l}\right)(1-\alpha) \frac{Y_{t}}{L_{t}} \\
\ln A_{t} & =(1-\rho) \ln (\bar{A})+\rho \ln A_{t-1}+\varepsilon_{t}
\end{aligned}
$$

Solving the model using the standard Blanchard and Kahn (1980) procedure, we can characterize approximate solutions to the model by using the standard log-linearization procedures. Defining the vectors $\widehat{\mathbf{x}}_{t}$ and $\widehat{\mathbf{s}}_{t}$ as the log-deviation of each variable:

$$
\widehat{\mathbf{x}}_{t}=\left[\begin{array}{c}
\widehat{y}_{t} \\
\hat{i}_{t} \\
\widehat{l}_{t} \\
\widehat{c}_{t}
\end{array}\right], \quad \widehat{\mathbf{s}}_{t}=\left[\begin{array}{l}
\widehat{k}_{t} \\
\widehat{a}_{t}
\end{array}\right]
$$

However, the resource constraint $Y_{t}=C_{t}+I_{t}$ holds by construction in the data. Thus, only the series for $\widehat{y}_{t}, \widehat{c}_{t}$ and $\widehat{l}_{t}$ are used in estimating the model as the series 
for $\widehat{i}_{t}$ is redundant. Therefore, $\widehat{\mathbf{x}}_{t}$ reduces to $3 \times 1$ vector with:

$$
\widehat{\mathbf{x}}_{t}=\left[\begin{array}{l}
\widehat{y}_{t} \\
\widehat{l}_{t} \\
\widehat{c}_{t}
\end{array}\right]
$$

The approximate solution of the model take the form:

$$
\begin{aligned}
\widehat{\mathbf{s}}_{t} & =\Gamma \widehat{\mathbf{s}}_{t-1}+\Xi \varepsilon_{t} \\
\widehat{\mathbf{x}}_{t} & =\Lambda \widehat{\mathbf{s}}_{t}
\end{aligned}
$$

where the elements of the matrices $\Gamma, \Xi$ and $\Lambda$ are function of the structural parameters of the model $\left(\alpha, \beta, \gamma, \delta, \rho, \sigma_{A}\right)$.

Note that the VAR representation of the DSGE model suffers from the stochastic singularity problem, given that the model has four observed variables and only one shock (the aggregate technology shock). The stochastic singularity problem has been dealt with in the literature first by introducing measurement errors and later by increasing the number of structural shocks. As pointed out by Ireland (2004), both alternatives have advantages but also disadvantages. The second approach is more consistent with the spirit of structural models and allows us to identify sources of aggregate fluctuations and to evaluate the contribution of each structural shock to the business cycle fluctuations. However, our focus is on evaluating forecasting performance and not on identifying sources of aggregate fluctuations. Additionally, we want to use the standard RBC model in order for the alternative methods (included the newly proposed one) to be comparable. Therefore, we consider the incorporation of measurement errors following Sargent (1989) and Ireland (2004), as explained below.

\subsection{The DSGE-AR method}

One approach to solving DSGE models is that proposed by Sargent (1989) and Altug (1989), by augmenting the model with unobservable errors. Following Sargent (1989) and Altug (1989), we add error terms to the observation equation (23). Therefore, we consider the following system:

$$
\begin{aligned}
\widehat{\mathbf{s}}_{t} & =\Gamma \widehat{\mathbf{s}}_{t-1}+\Xi \varepsilon_{t} \\
\widehat{\mathbf{x}}_{t} & =\Lambda \widehat{\mathbf{s}}_{t}+v_{t} \\
v_{t} & =\Theta v_{t-1}+\epsilon_{t}
\end{aligned}
$$

The matrix $\Theta$ is governing the persistence of the residuals, where its covariance matrix, $E_{t} \epsilon_{t} \epsilon_{t}^{\prime}=V$, is uncorrelated with the innovation to technology, $\varepsilon_{t}$.

Sargent (1989) and Altug (1989) assume that the measurement errors are uncorrelated with the data generated by the model, and thus, the matrices $\Theta$ and $V$ are diagonal, implying that the residual are uncorrelated across variables, 


$$
\begin{aligned}
& \Theta=\left[\begin{array}{ccc}
\theta_{y} & 0 & 0 \\
0 & \theta_{c} & 0 \\
0 & 0 & \theta_{l}
\end{array}\right] \\
& V=\left[\begin{array}{ccc}
v_{y}^{2} & 0 & 0 \\
0 & v_{c}^{2} & 0 \\
0 & 0 & v_{l}^{2}
\end{array}\right]
\end{aligned}
$$

Therefore, this method combines the DSGE model with an AR model for the measurement residuals. This approach has been applied by Altug (1989), McGrattan (1994), Ireland (2004), among others.

\subsection{The DSGE-VAR method}

Another possibility, proposed by Ireland (2004), is to consider a more general process for measurement errors, allowing the residuals to follow an unconstrained, first-order vector autoregression. As Ireland (2004) pointed out, this alternative has the advantage of imposing no restrictions on the cross-correlation of the measurement errors and thus capturing all movements and co-movements in the data not explained by the DSGE model. In this case, the matrices $\Theta$ and $V$ take the following form:

$$
\begin{array}{r}
\Theta=\left[\begin{array}{ccc}
\theta_{y} & \theta_{y c} & \theta_{y l} \\
\theta_{c y} & \theta_{c} & \theta_{c l} \\
\theta_{l y} & \theta_{l c} & \theta_{l}
\end{array}\right] \\
V=\left[\begin{array}{lll}
v_{y}^{2} & v_{y c} & v_{y l} \\
v_{c y} & v_{c}^{2} & v_{c l} \\
v_{l y} & v_{l c} & v_{l}^{2}
\end{array}\right]
\end{array}
$$

Ireland (2004) compare the forecasting performance of this method to the DSGEAR alternative approach of Sargent (1989), applied to the Hansen (1985) model for the US economy. Despite of the fact that his approach is more flexible and general in the treatment of the measurement errors, he finds that the forecast performance of the more restrictive DSGE-AR model outperforms the DSGE-VAR alternative.

\subsection{The VAR-DSGE approach}

A different strategy is derived from the method proposed by DeJong, Ingram and Whiteman (1993) and Ingram and Whiteman (1994), who use a Bayesian approach to estimate a DSGE model. ${ }^{5}$ Ingram and Whiteman (1994) compare the forecasting performance of a BVAR and a DSGE model over an unrestricted VAR and find as a result that the DSGE model is comparable to a Bayesian VAR with the Minnesota prior. This method has been further developed by Schorfheide (2000),

\footnotetext{
5 The original idea of this procedure is due to Doan et al. (1984) who propose shrinking vector autoregression coefficient estimates toward a prior view that vector times series are well-described as collections of independent random walks.
} 
Del Negro and Schorfheide (2004), Del Negro et al. (2007) by incorporating a prior from a DSGE model. The idea of the VAR-DSGE approach is to use prior information derived from DSGE model in the estimation of a VAR. DSGE models can be used to provide information about the parameters of a VAR. One possibility is to simulate data from the DSGE and to combine it with observed data and estimate a VAR, given a relative weight placed on the prior information. Therefore, this approach use the DSGE models only to set priors to a VAR.

The intuition behind this approach is that a DSGE model can be used to generate artificial data. A VAR can be estimated using both observed data and simulated data of the variables, in a certain proportion. That is, they propose estimating a VAR with an augmented data set of the observations and the artificial data generated by the DSGE model. The key parameter of this procedure is the weight, denoted by $\lambda$, placed on the DSGE models as the prior for the VAR, which may take values from zero to infinity. If the weight is large $(\lambda \rightarrow \infty)$, the resulting model will be close to the DSGE model itself and no weight is placed on the unrestricted VAR. If the weight is small $(\lambda \rightarrow 0)$, the resulting model will be close to an unrestricted VAR, with no weight on the DSGE model.

The procedure consists of estimating a VAR using both the actual and the artificial observations of the variables. We assume the following equation:

$$
Y=X \Phi+U
$$

where $Y$ is the vector of actual data, $X$ is the vector of regressors, including a constant, a trend and the lags of the variables and $U$ is a vector of VAR innovations.

The standard OLS estimator for $\Phi$ is given by:

$$
\Phi_{O L S}=\left(X^{\prime} X\right)^{-1} X^{\prime} Y
$$

The procedure considers $\lambda T$ artificial observations generated by a DSGE model for the variables of interest, where $\lambda$ is the weight of the prior. Using these artificial observations, the matrices $X^{*}$ and $Y^{*}$ are constructed. The VAR-DSGE procedure implies to estimating by OLS the augmented data set with both the actual and the artificial observations, which result in the following estimator:

$$
\Phi_{O L S}=\left(X^{\prime} X+X^{* \prime} X^{*}\right)^{-1}\left(X^{\prime} Y+X^{* \prime} Y^{* \prime}\right)
$$

Del Negro and Schorfheide (2004) report that for the US economy, the resulting model's out-of-sample forecasting outperforms a VAR. These authors show that even a relatively simple DSGE model used as a prior for a VAR is able to improve the forecasting performance relative to an unrestricted VAR. In this paper we apply this approach to the Spanish economy using the canonical RBC model described above. ${ }^{6}$

\footnotetext{
${ }^{6}$ The VAR-DSGE approach has been applied to the model with measurement errors. In order to check how results are affected by this procedure we also estimate a slightly extended version of the model with three additional structural shocks: a preference shock, a labour shock and an investment-specific technology shock. However, the forecasting performance of the VARD-DSGE approach with structural shocks does not change significantly.
} 


\section{A new method: the Augmented (B)VAR approach}

In this section we propose an alternative method to those mentioned above for taking DSGE models to the data, consisting on the expansion of the dimension of a VAR using sequences of artificial non-observed variables obtained from de DSGE model as auxiliary variables. The intuition is that a VAR model has limited information about the underlying dynamics of the variables, as opposed to the rich dynamics with which DSGE models are built. The procedure we propose seeks to exploit that richness by incorporating some of the unobserved variables delivered by the DSGE model. In practice, the procedure consists of estimating a standard VAR model or a Bayesian VAR model augmented with non-observable variables obtained from a DSGE model. In this case, the combination of the unrestricted VAR with the DSGE model is conducted by increasing the dimension of the VAR. ${ }^{7}$

Let $\mathbf{x}_{t}$ be a vector of observable economic variables assumed to drive the dynamics of the economy. The non-structural approach involves estimating a multivariate time series model using data for $\mathbf{x}_{t}$ alone. However, the structural approach assumes that DSGE models contain additional economic information, not fully captured by $\mathbf{x}_{t}$ that may be relevant to modeling the dynamics of these series. Let us suppose that this additional information can be summarized by a vector of (possibly unobserved) variables $\mathbf{z}_{t} . \mathbf{z}_{t}$ may be though as total factor productivity, marginal productivity, or any other information produced by the model that does not belong to the observed variable set.

We assume that the joint dynamics of $\left(\mathbf{x}_{t}, \mathbf{z}_{t}\right)$ are given by the following transition equation:

$$
\left[\begin{array}{c}
\mathbf{x}_{t} \\
\mathbf{z}_{t}
\end{array}\right]=\Phi(L)\left[\begin{array}{c}
\mathbf{x}_{t-1} \\
\mathbf{z}_{t-1}
\end{array}\right]+\left[\begin{array}{c}
\varepsilon_{t}^{x} \\
\varepsilon_{t}^{z}
\end{array}\right]
$$

where $\Phi(L)$ is a lag polynomial of infinite order $d$.

The above system can be interpreted as the reduced form of a DSGE model involving both observed and unobserved variables. This system reduces to a standard VAR in $\mathbf{x}_{t}$ if the terms of $\Phi(L)$ that relate $\mathbf{x}_{t}$ to $\mathbf{z}_{t-1}$ are all zero. But if the system (31) is the true system, the estimation of a VAR in $\mathbf{x}_{t}$ with the other information omitted will in general lead to biases estimates of the VAR coefficients. Note also that if non-observable variables contained in a DSGE model have information about the economy, then a natural solution to the degrees-of-freedom problem in VAR models is to use augmented standard VARs with estimation of those non-observable variables from a particular DSGE model.

The system (31) cannot be estimated directly because $\mathbf{z}_{t}$ are unobservable. However, $\mathbf{z}_{t}$ can be obtained through the use of a DSGE model. Let us provide an example how our method applies to the simple RBC model that we are using for comparisons. The example we present below is just one of the many ways in which one could try

\footnotetext{
7 Bernanke et al. (2005) proposed a similar method using a dynamic factor model to augment the VAR scale, the FAVAR (Factor-augmented vector autoregressive) model.
} 


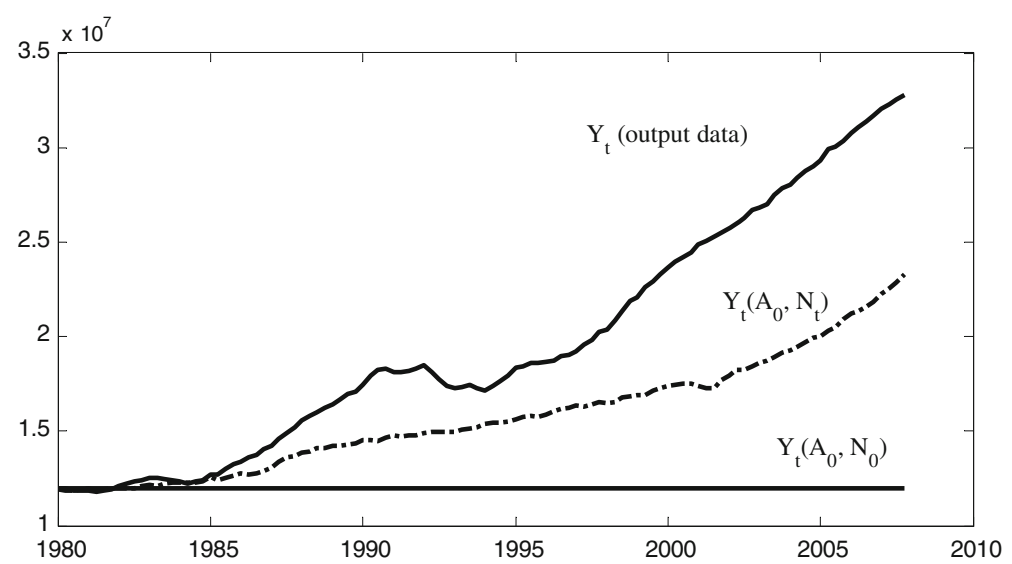

Fig. 1 Output with and without technical progress

to extract artificial data from the simple RBC model. The dimension of the vector of non-observed variables also depends on the particular DSGE model used.

In our simple artificial economy there are two sources of exogenous growth: one is given by population growth, and the other by exogenous technical change. We propose a method to separate the effects of these sources of growth using the DSGE model by creating a new variable $Z_{t}$, that will be used to expand the dimension of the VAR. To separate the endowment effect from technical change, we first calibrate the model to the level of observed output at the beginning of the sample as a steady state of the model. To do this, we use Newton's method to solve a system of equations where the parameters of the model are the variables and the variables are considered as parameters. Assume next that no technical change takes place during the sample time period. If, in addition, the population were constant, the economy would experience no variations from the calibration date. The sequence that would be observed starting from the calibration date onwards is shown in Fig. 1 as a flat line, $Y_{t}\left(A_{0}, N_{0}\right)$.

Assume now that only the population varies over time. The sequence that would be observed, according to the RBC model, starting from the calibration date onwards is shown in Fig. 1 as $Y_{t}\left(A_{0}, N_{t}\right)$. The change in total output can therefore be regarded as the variation induced by the change in the endowment of labour. Any remaining differences from actual output data, are interpreted as a residual that contains the exogenous technical change, and its effects on growth induced by that technical progress.

The ratio between the actual output and the no-technical progress output, denoted by $Z_{t}$ and shown in Fig. 2, is computed as $Z_{t}=Y_{t} / Y_{t}\left(A_{0}, N_{t}\right)$, the technical progress and the induced growth implied by the model. In the forecasting exercise, we incorporate the variable $Z_{t}$ generated from the model as an additional variable in the VAR with the key macroeconomic variables.

That is, we construct a VAR with the following specification:

$$
\left[\begin{array}{l}
\mathbf{x}_{t} \\
Z_{t}
\end{array}\right]=\left[\begin{array}{ll}
\phi_{11}(L) & \phi_{12}(L) \\
\phi_{21}(L) & \phi_{22}(L)
\end{array}\right]\left[\begin{array}{l}
\mathbf{x}_{t-1} \\
Z_{t-1}
\end{array}\right]+\left[\begin{array}{c}
\varepsilon_{t}^{x} \\
\varepsilon_{t}^{z}
\end{array}\right]
$$




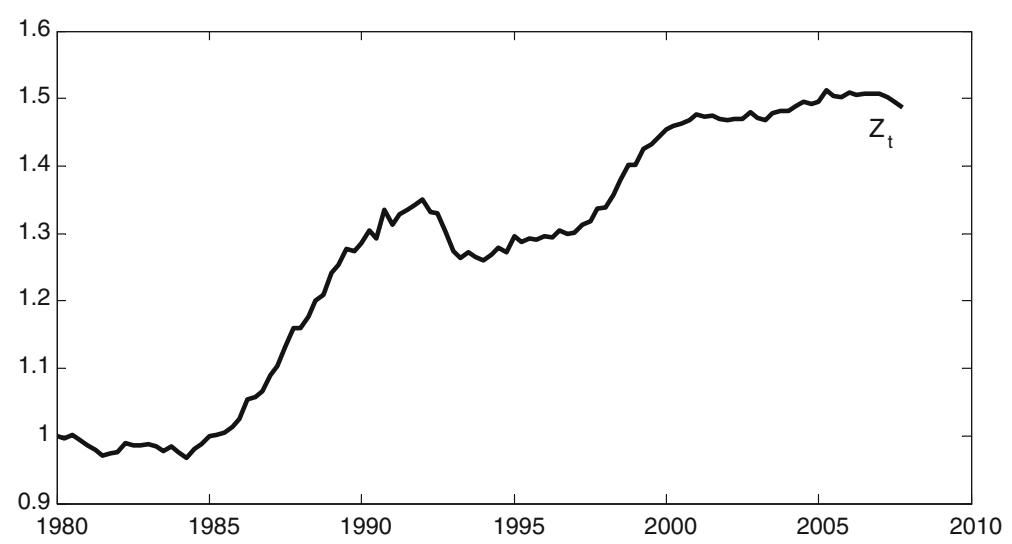

Fig. 2 Residual

where $\mathbf{x}_{t}$ are the macroeconomic data that the DSGE model seeks to explain and $Z_{t}$ is a vector derived from the DSGE model. If the model specification is correct, the relation between $\mathbf{x}_{t}$ and $Z_{t}$ should then capture additional economic information relevant to modelling the dynamics of $\mathbf{x}_{t}$. A standard unrestricted VAR implies that $\phi_{12}(L)=0$. Therefore, compared to a DSGE model the VAR omits relevant information and the model has implications for the observed variables as a function of the counterfactual. Thus, this method takes into account the information provided by the RBC model by augmenting the dimension of the VAR, adding one further non-observed variables reflecting technological progress. In the particular example shown above, we solve the RBC model assuming that, technical progress (the exogenous non-observable variable) is constant from the date of calibration onwards. The solution of the model provides the level of output that is consistent with that assumption. So we can obtain a measure of how technical progress would have affected output by comparing the actual level of output to that obtained from the model.

We have applied above the proposed method to a simple canonical RBC model with only one structural shock (i.e. a productivity shock). However, the idea behind the proposed new method for taking DSGE models to the data may be applied to any DSGE model. Note that the proposed method is not restricted to increasing the variable dimension of the VAR with only one additional non-observable variable generated through the model. Particularly, the number of no-observable variables obtained from the theoretical model will depend on the scale of the model and on the structural shocks added. This implies that the number of non-observable variables and the information included in them is a choice to be taken jointly with the choice of the specification of the model.

\section{Data, calibration and estimation}

\subsection{The data}

The analysis focuses on four key variables of the Spanish economy: output, consumption, investment and labor. The models are estimated for the Spanish economy based 
Table 1 Calibrated parameters

\begin{tabular}{lll}
\hline$\beta$ & Discount factor & 0.99 \\
$\delta$ & Depreciation rate & 0.025 \\
$\tau_{c}$ & Consumption tax & 0.09 \\
$\tau_{l}$ & Labor income tax & 0.33 \\
$\tau_{k}$ & Capital income tax & 0.22 \\
\hline
\end{tabular}

on seasonally-adjusted quarterly data on GDP, consumption, investment and hours worked for the period 1980:1-2007:4. Data are taken from the BD-REMS database. ${ }^{8}$ Labour is defined as full-time employment equivalent. Data are logged and linearly detrended for all models but the proposed AVAR approach.

\subsection{Calibration}

Before performing the estimation, we calibrate some parameters of the model. First, both the discount factor and the depreciation rate are set as they are difficult to estimate from the model in log-deviations from its steady-state. This is the standard strategy used in the literature, as estimated values lead to unreasonably low estimates of the discount factor and high estimates of the depreciation rate. The inter-temporal discount rate $\beta$ is set to 0.99 , which implies a steady state real interest rate of $4 \%$ and the depreciation rate $\delta$ is set to 0.025 .

Additionally, we keep the tax rates fixed. We use effective average tax rates, borrowed from Boscá et al. (2005). Table 1 summarizes the values for the calibrated parameters.

\subsection{Estimation}

The rest of the parameters are estimated using Bayesian methods. ${ }^{9}$ Fernández-Villaverde and Rubio-Ramírez (2004) argue that Bayesian estimates outperform maximum likelihood results. Therefore, instead of estimating the DSGE model via maximum likelihood, as in Altug (1989), McGrattan (1994) and Ireland (2004), the model is estimated using the Bayesian approach.

Prior distributions for structural parameters have been set by imposing plausible values, whereas for the measurement errors we assume flat priors. Specifically, for nonnegative parameters we assume Inverse Gamma prior distributions. For the parameters $\alpha, \gamma$ and $\rho$, we assume Beta prior distributions in order to keep them bounded between 0 and 1 . Finally, for the correlation of measurement residuals added to the model, we assume Uniform prior distributions with a range of $(-2,2)$.

Table 2 summarizes our assumptions regarding prior distributions for the estimated parameters and the posterior distribution corresponding to the DSGE-AR and DSGE-VAR models for the complete sample period. The estimates appear quite rea-

\footnotetext{
8 See Boscá et al. (2007) for a description of the BD-REMS database.

9 Estimates were drawn up using Dynare 4 running under Matlab v7, R14.
} 
Table 2 Prior and posterior distributions

\begin{tabular}{|c|c|c|c|c|c|c|c|}
\hline & \multirow{2}{*}{\multicolumn{3}{|c|}{ Prior distribution }} & \multicolumn{4}{|c|}{ Posterior distribution } \\
\hline & & & & \multicolumn{2}{|c|}{ DSGE-AR } & \multicolumn{2}{|c|}{ DSGE-VAR } \\
\hline & Distribution & Mean & SD/Range & Mean & SD & Mean & SD \\
\hline$\alpha$ & Beta & 0.35 & 0.1 & 0.2378 & 0.0068 & 0.2077 & 0.0059 \\
\hline$\gamma$ & Beta & 0.45 & 0.1 & 0.4903 & 0.0058 & 0.5066 & 0.0061 \\
\hline$\rho$ & Beta & 0.97 & 0.01 & 0.9996 & 0.0007 & 0.9998 & 0.0005 \\
\hline$\sigma_{A}$ & Inv. Gamma & 0.01 & Inf & 0.0101 & 0.0003 & 0.0079 & 0.0004 \\
\hline$\theta_{y}$ & Uniform & 0 & {$[-2,2]$} & 0.7850 & 0.086 & 0.7918 & 0.2263 \\
\hline$\theta_{c}$ & Uniform & 0 & {$[-2,2]$} & 0.5018 & 0.061 & 0.9871 & 0.1557 \\
\hline$\theta_{l}$ & Uniform & 0 & {$[-2,2]$} & 0.9993 & 0.023 & 0.9540 & 0.0636 \\
\hline$\theta_{y c}$ & Uniform & 0 & {$[-2,2]$} & - & - & -0.0174 & 0.0027 \\
\hline$\theta_{y l}$ & Uniform & 0 & {$[-2,2]$} & - & - & 0.0007 & 0.0001 \\
\hline$\theta_{c y}$ & Uniform & 0 & {$[-2,2]$} & - & - & -0.1397 & 0.0019 \\
\hline$\theta_{c l}$ & Uniform & 0 & {$[-2,2]$} & - & - & 0.0004 & 0.0000 \\
\hline$\theta_{l y}$ & Uniform & 0 & {$[-2,2]$} & - & - & -0.6540 & 0.0034 \\
\hline$\theta_{l c}$ & Uniform & 0 & {$[-2,2]$} & - & - & -0.6769 & 0.0046 \\
\hline$v_{y}$ & Inv. Gamma & 0.01 & Inf & 0.0074 & 0.0001 & 0.0018 & 0.0000 \\
\hline$v_{c}$ & Inv. Gamma & 0.01 & Inf & 0.0022 & 0.0000 & 0.0020 & 0.0001 \\
\hline$v_{l}$ & Inv. Gamma & 0.01 & Inf & 0.0068 & 0.0001 & 0.0147 & 0.0023 \\
\hline$v_{y c}$ & Inv. Gamma & 0.01 & Inf & - & - & 0.0076 & 0.0001 \\
\hline$v_{y l}$ & Inv. Gamma & 0.01 & Inf & - & - & 0.0042 & 0.0000 \\
\hline$v_{c l}$ & Inv. Gamma & 0.01 & Inf & - & - & 0.0053 & 0.0001 \\
\hline
\end{tabular}

sonable. However, the point estimates for the parameter $\alpha$ are relatively low $(0.237$ for the DSGE-AR model and 0.207 for the DSGE-VAR model).

\subsection{VAR models}

VARs models are widely used in macroeconomic forecasting. Moreover, DSGE models are easily represented through a VAR model. For these reasons, VARs models have been extensively used as a benchmark for evaluating the forecasting performance of alternative models, in particular, DSGE models.

One of the main advantage of VAR models is that they can be applied directly to the data, implying the existence of a relationship between each variable and past lagged values of all the variables considered in the model. One of the drawbacks of VARs is the problem of overfitting, which results in inefficient estimates and large out-of-sample forecasting errors. Unrestricted VARs models may have too may parameters, and thus, the estimates may be very imprecise, specially in small samples. This is particularly important when we consider longer forecasting horizons, as VAR forecasting performance deteriorates rapidly. The problem of overfitting with standard VARs can be overcome using Bayesian methods. The Bayesian VAR analysis, 
developed by Litterman (1981), imposes restrictions on some coefficients by introducing prior information, generating more precise parameter estimates.

In our analysis we estimate VARs and Bayesian VARs for the four macroeconomics variables (output, consumption, investment and labor) of the Spanish economy contained in the DSGE model as a benchmark. Our basic VAR model includes a constant and a trend term. The VAR is estimated using one lag of the variables. The BVAR specification chosen is a VAR with the Minnesota prior.

\section{Forecast evaluation}

This section analyses the out-of-sample performance of the competing models over the four key macroeconomic variables for the Spanish economy. There is a relatively large number of recent papers that compare the forecasting performance of DSGE and VAR models, such as Smets and Wouters (2004), Ireland (2004), Del Negro et al. (2007), Adolfson et al. (2007), Christoffel et al. (2008), Rubaszek and Skrzypczynski (2008), among others. In general, they find that the use of DSGE models improves forecasting performance compared with VAR methods.

We report the out-of-sample forecast performance of seven different alternative models: VAR, BVAR, DSGE-AR, DSGE-VAR, VAR-DSGE, AVAR and ABVAR models. The out-of-sample forecast analysis is performed for horizons ranging from one up to eight quarters ahead. The forecast accuracy evaluation period is 1995:12007:4. Therefore, all the models are estimated initially over the first 60 periods (1980:1 through 1994:4). These estimations are used to generate forecasts for the period 1995:1-1996:4. The model is then re-estimated over 61 periods, incorporating one additional observations, 1980:1-1995:1, and the forecasts are recalculated for the period 1995:2-1997:1, and so on until the end of the sample period. This procedure implies that the one period ahead forecast is calculated from 1995:1 to 2007:4. The two period ahead forecast is calculated for the period 1995:2-2007:4, and so on. Therefore, each model is re-estimated 52 times.

The forecasting performance of the competing models is evaluated along two dimensions: the bias in errors and the absolute size of errors. The bias in errors is measured by the mean absolute error (MAE), while the absolute size of errors is measured by the root-mean squared error (RMSE). Table 3 summarizes the results for the MAE statistics for the bias errors. The best results are generated by the DSGE-AR and the ABVAR models. For all the four variables, the ABVAR model outperforms all other alternatives for one period ahead forecasts. The ABVAR model is also superior forecasting investment in all periods. However, for the other three variables, output, consumption and labour, the forecasting performance of the DSGE-AR model outperforms those of the other alternatives with the exception of one period ahead, where ABVAR is superior. In general, we find that the results from the DSGE-AR model and the ABVAR model are comparable, with these being the alternatives that produce the most accurate forecasts.

Table 4 summarizes the results in terms of the root-mean squared error. The results are similar to those obtained for the MAE statistic. First, we obtain that the forecasting performance of an AVAR is superior to that of an unrestricted VAR for all the 
Table 3 Forecasting MAE

\begin{tabular}{|c|c|c|c|c|c|c|c|c|}
\hline & \multicolumn{8}{|c|}{ Periods ahead } \\
\hline & 1 & 2 & 3 & 4 & 5 & 6 & 7 & 8 \\
\hline \multicolumn{9}{|l|}{ Output } \\
\hline VAR & 0.607 & 1.255 & 1.950 & 2.675 & 3.408 & 4.132 & 4.830 & 5.486 \\
\hline BVAR & 0.155 & 0.305 & 0.452 & 0.594 & 0.734 & 0.871 & 0.983 & 1.085 \\
\hline DSGE-AR & 0.154 & 0.256 & 0.347 & 0.437 & 0.527 & 0.612 & 0.681 & 0.748 \\
\hline DSGE-VAR & 0.320 & 1.829 & 2.841 & 3.604 & 4.105 & 4.497 & 4.476 & 4.467 \\
\hline VAR-DSGE & 1.530 & 1.614 & 2.270 & 3.309 & 4.454 & 5.617 & 5.653 & 5.690 \\
\hline AVAR & 0.553 & 0.517 & 1.216 & 1.513 & 2.167 & 2.914 & 3.759 & 4.676 \\
\hline ABVAR & 0.120 & 0.268 & 0.409 & 0.547 & 0.685 & 0.817 & 0.930 & 1.040 \\
\hline \multicolumn{9}{|l|}{ Consumption } \\
\hline VAR & 0.499 & 1.024 & 1.581 & 2.163 & 2.763 & 3.365 & 3.954 & 4.523 \\
\hline BVAR & 0.136 & 0.267 & 0.398 & 0.531 & 0.670 & 0.805 & 0.902 & 0.993 \\
\hline DSGE-AR & 0.229 & 0.287 & 0.431 & 0.482 & 0.605 & 0.660 & 0.754 & 0.835 \\
\hline DSGE-VAR & 0.179 & 1.831 & 2.867 & 3.561 & 3.980 & 4.283 & 4.277 & 4.284 \\
\hline VAR-DSGE & 1.381 & 1.649 & 1.976 & 2.389 & 2.845 & 3.325 & 3.323 & 3.325 \\
\hline AVAR & 0.496 & 0.358 & 0.718 & 0.850 & 1.281 & 1.761 & 2.295 & 2.887 \\
\hline ABVAR & 0.109 & 0.243 & 0.375 & 0.509 & 0.644 & 0.778 & 0.877 & 0.971 \\
\hline \multicolumn{9}{|l|}{ Investment } \\
\hline VAR & 0.415 & 0.808 & 1.181 & 1.539 & 1.898 & 2.244 & 2.586 & 3.022 \\
\hline BVAR & 0.130 & 0.252 & 0.358 & 0.458 & 0.564 & 0.668 & 0.769 & 0.864 \\
\hline DSGE-AR & 1.091 & 1.070 & 1.083 & 1.085 & 1.081 & 1.116 & 1.138 & 1.172 \\
\hline DSGE-VAR & 1.282 & 1.339 & 1.435 & 1.508 & 1.612 & 1.688 & 1.721 & 1.751 \\
\hline VAR-DSGE & 1.328 & 1.067 & 1.367 & 1.929 & 2.593 & 3.233 & 3.236 & 3.231 \\
\hline AVAR & 0.440 & 1.579 & 2.923 & 4.013 & 5.177 & 6.326 & 7.481 & 8.658 \\
\hline ABVAR & 0.092 & 0.210 & 0.317 & 0.420 & 0.527 & 0.632 & 0.731 & 0.826 \\
\hline \multicolumn{9}{|l|}{ Labour } \\
\hline VAR & 0.735 & 1.513 & 2.324 & 3.154 & 3.990 & 4.831 & 5.667 & 6.481 \\
\hline BVAR & 0.215 & 0.432 & 0.654 & 0.879 & 1.107 & 1.334 & 1.502 & 1.666 \\
\hline DSGE-AR & 0.196 & 0.375 & 0.540 & 0.693 & 0.833 & 0.966 & 1.098 & 1.231 \\
\hline DSGE-VAR & 8.284 & 9.755 & 10.440 & 10.692 & 10.928 & 11.112 & 10.951 & 10.795 \\
\hline VAR-DSGE & 18.059 & 18.433 & 18.775 & 19.089 & 19.376 & 19.640 & 19.756 & 19.870 \\
\hline AVAR & 0.712 & 0.666 & 0.845 & 0.878 & 1.025 & 1.224 & 1.483 & 1.785 \\
\hline ABVAR & 0.179 & 0.397 & 0.618 & 0.841 & 1.066 & 1.294 & 1.462 & 1.626 \\
\hline
\end{tabular}

Numbers in boldface indicate the lower MAE

variables and for all forecast horizons, with the sole exception of investment, where this relationship is inverted. More remarkable is the absolute superiority of the forecasting performance of the ABVAR model compared to the BVAR model. Note that the Augmented (B)VAR models nest standard (B)VAR models, so they are directly comparable. This comparison reveals that augmenting the number of variables in the 
Table 4 Forecasting RMSE

\begin{tabular}{|c|c|c|c|c|c|c|c|c|}
\hline & \multicolumn{8}{|c|}{ Periods ahead } \\
\hline & 1 & 2 & 3 & 4 & 5 & 6 & 7 & 8 \\
\hline \multicolumn{9}{|l|}{ Output } \\
\hline VAR & 0.674 & 1.386 & 2.127 & 2.883 & 3.722 & 4.488 & 5.229 & 5.931 \\
\hline BVAR & 0.252 & 0.532 & 0.816 & 1.098 & 1.379 & 1.654 & 1.864 & 2.056 \\
\hline DSGE-AR & 0.435 & 0.628 & 0.773 & 0.898 & 1.022 & 1.145 & 1.249 & 1.356 \\
\hline DSGE-VAR & 1.662 & 4.501 & 6.722 & 8.349 & 9.394 & 10.230 & 10.122 & 10.032 \\
\hline VAR-DSGE & 2.897 & 2.947 & 4.399 & 6.228 & 8.092 & 9.915 & 9.930 & 9.952 \\
\hline AVAR & 0.627 & 0.681 & 0.946 & 1.904 & 2.702 & 3.599 & 4.586 & 5.658 \\
\hline ABVAR & 0.249 & 0.515 & 0.780 & 1.044 & 1.302 & 1.550 & 1.755 & 1.944 \\
\hline \multicolumn{9}{|l|}{ Consumption } \\
\hline VAR & 0.551 & 1.121 & 1.708 & 2.311 & 3.004 & 3.636 & 4.259 & 4.861 \\
\hline BVAR & 0.229 & 0.482 & 0.735 & 0.987 & 1.238 & 1.488 & 1.666 & 1.834 \\
\hline DSGE-AR & 0.733 & 0.585 & 0.964 & 0.932 & 1.213 & 1.225 & 1.369 & 1.503 \\
\hline DSGE-VAR & 0.536 & 4.016 & 6.132 & 7.507 & 8.228 & 8.732 & 8.634 & 8.554 \\
\hline VAR-DSGE & 2.847 & 3.310 & 3.823 & 4.409 & 5.054 & 5.746 & 5.765 & 5.789 \\
\hline AVAR & 0.555 & 0.440 & 0.475 & 1.149 & 1.650 & 2.214 & 2.836 & 3.519 \\
\hline ABVAR & 0.233 & 0.483 & 0.731 & 0.978 & 1.222 & 1.461 & 1.637 & 1.804 \\
\hline \multicolumn{9}{|l|}{ Investment } \\
\hline VAR & 0.600 & 1.128 & 1.586 & 1.997 & 2.377 & 2.811 & 3.256 & 3.723 \\
\hline BVAR & 0.198 & 0.399 & 0.592 & 0.768 & 0.938 & 1.104 & 1.279 & 1.451 \\
\hline DSGE-AR & 1.907 & 1.920 & 1.958 & 1.974 & 1.988 & 2.030 & 2.047 & 2.062 \\
\hline DSGE-VAR & 2.437 & 2.504 & 2.945 & 3.359 & 3.773 & 4.114 & 4.091 & 4.077 \\
\hline VAR-DSGE & 2.751 & 2.138 & 2.657 & 2.664 & 4.722 & 5.710 & 5.668 & 5.637 \\
\hline AVAR & 0.564 & 1.699 & 2.817 & 4.122 & 5.300 & 6.478 & 7.678 & 8.918 \\
\hline ABVAR & 0.192 & 0.388 & 0.581 & 0.757 & 0.925 & 1.094 & 1.268 & 1.441 \\
\hline \multicolumn{9}{|l|}{ Labour } \\
\hline VAR & 0.775 & 1.585 & 2.416 & 3.257 & 4.206 & 5.064 & 5.913 & 6.742 \\
\hline BVAR & 0.320 & 0.683 & 1.050 & 1.420 & 1.794 & 2.170 & 2.441 & 2.705 \\
\hline DSGE-AR & 0.583 & 0.982 & 1.297 & 1.569 & 1.812 & 2.037 & 2.195 & 2.368 \\
\hline DSGE-VAR & 15.682 & 17.997 & 18.919 & 19.149 & 19.390 & 19.557 & 19.269 & 18.997 \\
\hline VAR-DSGE & 28.119 & 28.510 & 28.855 & 29.157 & 29.422 & 29.652 & 29.668 & 29.684 \\
\hline AVAR & 0.771 & 0.762 & 0.757 & 1.004 & 1.234 & 1.520 & 1.850 & 2.224 \\
\hline ABVAR & 0.329 & 0.685 & 1.046 & 1.409 & 1.773 & 2.136 & 2.406 & 2.669 \\
\hline
\end{tabular}

Numbers in boldface indicate the lower RMSE

VAR with non-observable variables obtained from a DSGE model incorporates useful information for forecasting. For output, the best alternative is the DSGE-AR for longer horizons while for one period ahead the ABVAR alternative produces better results. For consumption the results are mixed, as the DSGE-AR model and the ABVAR model produces very similar RMSE estimates, with the lowest being those produced 
by ABVAR from the one to three periods ahead forecast. For investment, the ABVAR model is again the best alternative for all horizons. For employment the forecasting performance of the AVAR model outperforms all other DSGE-based alternative and it is only challenged by the BVAR model forecasts for one and two periods ahead. DSGE-VAR and VAR-DSGE models display relatively large RMSE values, specially for output and labour. It is noticeable than the forecasting performance of the DSGEVAR is worse than that of the DSGE-AR. Ireland (2004) also obtains a similar result when comparing the forecasting performance of the two alternatives estimated by maximum likelihood.

From the results summarized in Tables 3 and 4 we can highlight the following preliminary conclusions. First, we find that BVAR methods are superior to unrestricted VARs for all horizons, confirming previous analysis. Second, RMSE increases at a higher rate in the case of a VAR than in a BVAR, This implies that Bayesian methods are clearly superior for forecasting at longer horizons. Third, the DSGE-AR model outperforms the DSGE-VAR model, confirming the results obtained by Ireland (2004). Finally, the results show that the method proposed in this paper, the Augmented VAR model, can be very useful in macroeconomic forecasting, and that it is superior at least in some dimensions to the alternatives. The AVAR model outperforms the VAR and the ABVAR model outperforms the BVAR in all cases. The only alternative with a similar forecasting performance accuracy is the DSGE-AR model, which is superior for output and labour, and consumption for longer time horizons. The analysis conducted in this paper confirms previous results, highlighting that DSGE models with a deep theoretical background, combined with the flexibility of the standard VAR approach, can be a very useful tool in macroeconomic forecasting.

\section{Conclusions}

In this paper we propose a new approach to combining DSGE and VAR models. The proposed method is different from existing methods and consists of augmenting the space of the VAR with non-observables variables artificially generated by a DSGE model. The intuition behind our proposal is that DSGE models contain additional information about the underlying dynamics of actual data and that this information can be incorporated in an otherwise standard VAR model.

The results obtained from the forecasting exercise conducted for the Spanish economy show that the proposed Augmented (B)VAR model is superior to the alternative approaches in forecasting key macroeconomic variables of the economy being only challenged by the DSGE-AR model. We wish to stress that our method establishes a metric for comparing the forecasting performance of a DSGE model with other non-structural methods. Given that A(B)VAR models encompass their (B)VAR counterparts, we can attribute all the gains in forecasting accuracy to the artificial variables obtained from the DSGE model. What we have shown in the paper is just a simple example of what could be done with a larger scale DSGE model. It would be possible to assess the relative improvement in forecasting accuracy gained from augmenting the VAR with the additional variables derived from the larger scale DSGE model. 
All the analysis conducted in the paper have been carried out using a very simple DSGE model. The choice of a small scale model is not random: it serves to check whether even a simple DSGE model specification can be useful as a forecasting tool. A natural extension to our work would be to consider richer DSGE models, in order to asses how the forecasting performance of DSGE models is related to the their scale. In principle, we would expect that forecasting performance of the DSGE models will increase with the scale. The power of DSGE models incorporating New Keynesian features to capture some salient features found in macroeconomic data has been successfully exploited by recent literature, improving our understanding of economic dynamics. In this sense, it would be of particular interest to apply the above analysis to the current workshorse New Keynesian DSGE model. The study of the forecasting performance of alternative more complex DSGE models with different size is part our current research agenda. Finally, our analysis supports the view, consistent with previous literature, that DSGE models are useful to policymakers for forecasting macroeconomic variables.

Open Access This article is distributed under the terms of the Creative Commons Attribution License which permits any use, distribution and reproduction in any medium, provided the original author(s) and source are credited.

\section{References}

Adolfson M, Laséen S, Lindé J, Villani M (2007) RAMSES, a new general equilibrium model for monetary policy analysis. Riksbank Econ Rev 2:5-39

Altug S (1989) Time-to-build and aggregate fluctuations: Some new evidence. Int Econ Rev 30(4):889-920

Andrés L, Burriel P, Estrada A (2006) BEMOD: a DSGE model for the Spanish economy and the rest of the Euro Area. Banco de España working paper, no. 631

Bernanke B, Boivin J, Eliasz P (2005) Measuring the effects of monetary polity: a factor-augmented vector autoregressive (FAVAR) approach. Q J Econ 120(1):387-422

Blanchard J, Kahn C (1980) The solution of linear difference models under rational expectations. Econometrica 28(5):1305-1311

Boscá J, García J, Taguas D (2005) Taxation in the OECD: 1965-2001. Working Paper Ministerio de Economía y Hacienda, D-2005-06

Boscá J, Díaz A, Doménech R, Ferri J, Pérez E, Puch L (2007) The REMSDB macroeconomic database of the Spanish economy. Mimeo

Boscá J, Díaz A, Doménech R, Ferri J, Pérez E, Puch L (2009) A rational expectations model for simulation and policy evaluation of the Spanish economy. SERIEs 1(1-2):135-169

Burriel P, Fernández-Villaverde J, Rubio-Ramírez J (2009) MEDEA: a DSGE model for the Spanish economy. SERIEs 1(1-2):175-243

Canova F (2002) Validating monetary DSGE models through VARs. Mimeo

Christiano L, Eichenbaum M, Evans C (2005) Nominal rigidities and the dynamic effects to a shock of monetary policy. J Political Econ 113(1):1-45

Christoffel K, Coenen G, Warne A (2008) The new area-wide model of the euro area-a micro-founded open-economy model for forecasting and policy analysis. European Central Bank Working Paper Series, no. 944

DeJong D, Ingram B, Whiteman C, (1993) Evaluating VARs with Monetary Business Cycle Priors. Proceedings of the American Statistical Association, Bayesian Statistical Science Section, pp 160-169

Del Negro M, Schorfheide F (2003) Take your model bowling: forecasting with general equilibrium models. Federal Reserve Bank of Atlanta Econ Rev Q4:35-50

Del Negro M, Schorfheide F (2004) Priors from general equilibrium models for VARs'. Int Econ Rev 45(2):643-673 
Del Negro M, Schorfheide F, Smets F, Wouters R (2007) On the fit of new Keynesian models. J Bus Econ Stat 25(2):123-143

Diebold F (1998) The past, present and future of macroeconomic forecasting. J Econ Perspect 12(2):175192

Doan T, Litterman R, Sims C (1984) Forecasting and conditional projection using realistic prior distributions. Econometric Reviews 3(1):1-100

Edge R, Kiley M, Laforte J (2008) Natural rate measures in an estimated DSGE model of the US economy. J Econ Dyn Control 32(8):2512-2535

Erceg C, Guerrieri L, Gust C (2006) SIGMA: a new open economy model for policy analysis. Int J Central Bank 2(1):1-50

Fernández-Villaverde J, Rubio-Ramírez JF (2004) Comparing dynamic equilibrium models to data: a Bayesian approach. J Econom 123:153-187

Hansen G (1985) Indivisible labor and the business cycle. J Monet Econ 16(3):309-327

Ingram B, Whiteman C (1994) Supplanting the "Minnesota" priors. Forecasting macroeconomic time series using real business cycle model priors. J Monet Econ 34(4):497-510

Ireland P (2004) A method for taking models to the data. J Econ Dyn Control 28:1205-1226

Litterman R, (1981) A Bayesian procedure for forecasting with vector autoregressions. Working Paper, Federal Reserve Bank of Minneapolis

Litterman (1986a) A statistical approach to economic forecasting. J Bus Econ Stat 4(1):1-4

Litterman (1986b) Forecasting with Bayesian vector autoregressions: five years of experience. J Bus Econ Stat 4(1):25-38

Lucas R (1976) Econometric policy evaluation: a critique. Carnegie Rochester Conf Ser Public Policy 1:9-46

McGrattan E (1994) The macroeconomic effects of distortionary taxation. J Monet Econ 33:573-601

Sargent T (1989) Two models of measurements and the investment accelerator. J Political Econ 97(2):251287

Schorfheide F (2000) Loss function-based evaluation of DSGE models. J Appl Econom 15(6):645-670

Sims C (1980) Macroeconomics and reality. Econometrica 48(1):1-48

Smets F, Wouters R (2003) An estimated dynamic stochastic general equilibrium model for the Euro area. J Eur Econ Assoc 1(5):1123-1175

Smets F, Wouters R (2004) Forecasting with a Bayesian DSGE Model: An Application to the Euro Area. Journal of Common Market Studies 42(4):841-867

Smets F, Wouters R (2005) Comparing shocks and frictions in US and Euro business cycles: a Bayesian DSGE approach. J Appl Econom 20(2):161-183

Rubaszek M, Skrzypczynski P (2008) On the forecasting performance of a small-scale DSGE model. Int J Forecast 24:498-512 